\title{
'It's a Bit Weird, but it's OK'? How Female Computer Science Students Navigate Being a Minority
}

\author{
Emily Winter \\ Lancaster University \\ Lancaster, UK
}

\author{
Lisa Thomas \\ Lancaster University \\ Lancaster, UK
}

\author{
Lynne Blair \\ Lancaster University \\ Lancaster, UK
}

\begin{abstract}
Within Computer Science (CS) education, women have long been underrepresented. In the UK, women make up less than $20 \%$ of CS students at A-Level and undergraduate level. The lack of diversity within CS has become well-studied, often through quantitative surveys of female CS students or evaluations of different pedagogical or cultural interventions aimed to increase diversity and inclusivity. However, there have been far fewer studies that explore the experiences of female CS students at a more in-depth, qualitative level. This paper reports on the results of 15 in-depth semi-structured interviews with first year female CS undergraduate students at a UK university. Several of our findings are consistent with much existing research, such as the prevalence of gendered CS stereotypes and a lack of confidence. However, we also find two key strategies by which female Computer Science students navigate their minority experience that have not been given much prior attention. Firstly, we find that female CS students find it hard to articulate their minority experience without utilising the linguistic device of hedging. Asked about their experiences as a minority, participants would often mention a negative feeling in relation to this situation, followed quickly by a diminishing clause- 'it's a bit weird, but it's ok'. Secondly, participants tended to individualise the problem, stressing the importance of their own individual responsibility to fit into CS and succeed, despite having sometimes experienced discrimination or sexism. We conclude by considering the implications of these findings for educators.
\end{abstract}

\section{CCS CONCEPTS}

- Social and professional topics $\rightarrow$ Computing education.

\section{KEYWORDS}

Computing education, diversity, gender, qualitative research

\section{ACM Reference Format:}

Emily Winter, Lisa Thomas, and Lynne Blair. 2021. 'It's a Bit Weird, but it's OK'? How Female Computer Science Students Navigate Being a Minority. In 26th ACM Conference on Innovation and Technology in Computer Science Education V. 1 (ITiCSE 2021), June 26-fuly 1, 2021, Virtual Event, Germany. ACM, New York, NY, USA, 7 pages. https://doi.org/10.1145/3430665.3456329

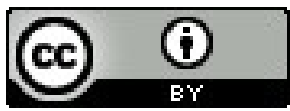

This work is licensed under a Creative Commons Attribution International 4.0 License. ITiCSE 2021, fune 26-fuly 1, 2021, Virtual Event, Germany. (C) 2021 Copyright held by the owner/author(s). ACM ISBN 978-1-4503-8214-4/21/06

https://doi.org/10.1145/3430665.3456329

\section{INTRODUCTION}

A considerable gender imbalance is a marked feature of computer science (CS), both in educational contexts and within CS-related workplaces. Women made up $14.4 \%$ of those in the UK that sat a CS A-Level ${ }^{1}$ in 2020 [13] and $18.4 \%$ of those who started a CS-related degree at a UK university in 2018 [16]. The percentage of women IT specialists in the UK is similarly low, just $17 \%$ in 2019 [4]. This situation is of concern for many reasons. Firstly, this gender imbalance has a negative effect on women who enter the field, from feeling uncomfortable to experiences of sexism, stereotyping or alienating macho work cultures. The State of European Tech survey in 2020 found that $42 \%$ of female respondents had experienced some form of discrimination [2]. Secondly, the image of CS as a male field may put off otherwise interested and competent women from entering in the first place. Thirdly, there are a range of broader issues regarding the societal implications of technologies being made predominantly by men, and the (un)conscious biases that may become embedded in technology design and development as a result. High profile examples of this include gender stereotypes in Google translate [17]; voice-recognition software that is better at recognising male voices; and the release of Apple's health monitoring system in 2014 without a menstrual cycle tracker [25].

In this paper, we explore the experiences of first year female CS undergraduates, specifically focusing on their sense of belonging and how they navigate being a visible minority. Whilst the topic of diversity in CS has been given much attention, far less research has explored, through in-depth qualitative research, the lived experiences of women within CS.

The paper is structured as follows: Section 2 identifies related work, situating our own approach. Section 3 presents our research questions and contributions; Section 4 our methodology; and Section 5 our findings. We discuss the implications of these findings for CS educators in Section 6, concluding by identifying directions for future research in Section 7.

\section{RELATED WORK}

There is a large literature related to diversity in CS, particularly gender diversity. Within this literature, two key tendencies can be identified. Firstly, there is a lot of work that explores CS student experiences, and gender differences, by means of quantitative survey-based research (for example, [1] [6] [20] [23] [27] [29] [34] [36]). Secondly, there is research that evaluates the impact of different kinds of pedagogical or cultural interventions for encouraging women's participation in CS (for example, [3] [15] [30] [40]).

Our work can be situated within a small body of qualitative research in this field. Qualitative approaches are not common in

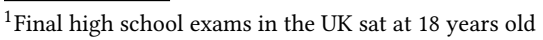


studies of diversity in CS education, despite the use of qualitative interviews by Margolis and Fisher in Unlocking the Clubhouse [21], which was published in 2002 and has been highly influential. In a recent article, however, Collain and Trytten [9] caution against the findings reported in Unlocking the Clubhouse being seen as universal, drawing attention to the changes that have occurred since its publication and the 'exceptional' status of the university where the research was conducted, Carnegie Mellon, in terms of its international prestige. Collain and Trytten suggest that qualitative research at other universities is important for gaining insights from higher education institutions that may be more representative. Whilst our own institution is highly ranked within the UK, it also attracts students from a wide range of socio-economic backgrounds. Of the 2016/17 intake of students, 33\% were the first in their family to attend university. This compares with $9 \%$ first generation college students in Carnegie Mellon University's most recent intake.

Some other recent work has taken a qualitative, interview-based approach. Our work has particular similarities with Rankin and Thomas's qualitative research into the 'lived experiences' of black women in CS [28] and with Falkner et al.'s interviews with female $\mathrm{PhD}$ students and academics in CS in Australia, exploring 'the thoughts and perceptions of high-performing women who have stayed in Computer Science academia' [12]. Whilst our participants represent a different demographic (first year female undergraduate students), like both these recent publications we focus on women's strategies for navigating their experience of being in the minority. Our work responds to literature that has highlighted the lack of research into women's affective experiences in CS [6].

A key conceptual framework for our research is the notion of a 'sense of belonging'. Within social psychology, there is some work that has considered academic belonging, Walton and Cohen's work on race being particularly influential. Walton and Cohen coin the term 'belonging uncertainty' to describe how 'in academic and professional settings, members of socially stigmatised groups are more uncertain of the quality of their social bonds and thus more sensitive to issues of social belonging' [38]. This 'belonging uncertainty' can undermine motivation and achievement. Research has also explored belonging in STEM specifically, with Smith highlighting that a sense of exerting more effort than others can lead to a decreased sense of belonging among women in STEM [35] and Lewis identifying that members of underrepresented groups experience greater threats to their sense of belonging [19].

The sense of belonging concept has also been applied within studies of diversity in CS specifically, for example Stout et al.'s study of the impact of formal research experiences upon female CS students' sense of belonging [36]. Stout et al. define sense of belonging as 'the subjective feeling of fitting in and being involved as a valued and legitimate member in a particular setting'. Furthermore, sense of belonging is 'a robust predictor of academic motivation, engagement and achievement in education settings'. Mishkin's work stresses that 'for young women in technology, an educational minority, sense of belonging is both a necessary condition of motivation and is a significantly stronger predictor of motivation than is autonomy or competency' [23]. She continues: 'to feel efficacious enough to pursue computing, women need to feel a sense of belonging'. Given its implications for motivation and success in CS, a sense of belonging was a key concept that we explored in our research.

\section{RESEARCH QUESTIONS AND CONTRIBUTION}

The aim of this paper is to provide an in-depth, qualitative exploration of the experiences of female CS undergraduate students at our institution. Our research questions are as follows:

- RQ1: What are the experiences of female CS undergraduate students?

- RQ2: How do female CS undergraduate students interpret and navigate their status as a minority?

- RQ3: Do female CS undergraduate students feel a sense of belonging?

Our main contribution is to offer two key findings related to female CS undergraduate students' experiences, their sense of belonging, and their negotiation of being a minority. Firstly, we find a great deal of uncertainty in how female students articulate their minority status and negative experiences. The linguistic device of hedging was commonly used to diminish more uncomfortable experiences. This demonstrates the fragility of these young women's sense of belonging, with effort needed to mitigate negative circumstances. Secondly, we find that female CS students often individualise the problem. Whilst decades of CS education research has unpicked various complex, structural reasons for the lack of gender diversity in CS, our participants stressed that the responsibility to succeed and fit in was their own personal, individual responsibility. This is a key strategy by which young women navigate being a minority in CS and demonstrates a strong sense of personal agency, but we suggest that this individualisation may also have negative impacts. We conclude our discussion of these findings (Section 5) by identifying some implications for educators (Section 6) and directions for future research (Section 7).

\section{METHODOLOGY}

Semi-structured, in-depth interviews were carried out with firstyear female students who were studying CS as their major, as part of a joint degree, or as their minor. We chose to interview students in the middle of the first term of their first academic year, because we wanted to hear about their early experiences of university-level CS. Information about the study was disseminated through lectures and labs. It was made clear that the interviews were about women's experiences within CS and that participation was voluntary. Being entered into a prize draw for an online gift voucher was offered as an incentive. Interested students provided their email address, resulting in 15 interviews. Two participants were taking CS as a minor; the rest were either majoring in CS (7 participants) or studying CS as part of a joint programme (6 participants). The participants' prior CS experience varied considerably. 6 participants had studied CS at A Level (with another participant studying the A Level for one year); 2 had studied IT at A Level. Three had studied $\mathrm{GCSE}^{2} \mathrm{CS}$. The remaining four, aside from IT at GCSE, only had informal CS learning experiences.

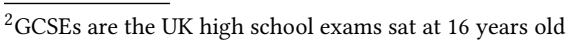


A sample size of fifteen is similar to other qualitative research in this area [12] [28]. In addition, the thirteen participants who were either majoring in CS or studying it as part of a joint degree represent $26 \%$ of the female first year cohort. This is a good volunteer sample for an interview study, where participants were asked for an hour of their time and attended an interview on campus.

Interviews were semi-structured and questions were asked around the following key topics: schooling and early experiences of computing; choice of major and minor; current experiences of CS and expectations for the future; feelings around the gender gap; and perceptions of CS. Interviews also involved a card ranking exercise where participants were asked to rank a series of statements according to their level of agreement onto a paper grid with five boxes, ranging from 'strongly agree' to 'strongly disagree'. This exercise was not designed to yield quantitative data but rather to elicit responses to a range of statements related to sense of belonging. Table 1 shows these statements and their connection to nine thematic categories that we identified from the literature as contributing to women's sense of belonging within CS.

This study had the approval of the authors' university's research ethics committee. All participation was voluntary and involved informed consent. The voluntary nature of study participation was particularly stressed, given that participants were students, with care being taken to emphasise that (non-)participation would not have any impact on their grades. The interviewer was a member of staff with no teaching responsibilities at the time. All participants were given the option to view their transcript if they wished, or to identify anything that they had said as 'off the record' and not to be quoted in any publication. We have taken care to remove any information that could potentially identify participants, with all key participant information provided only in aggregated form. To protect participant anonymity, we also do not report other key demographics of our participants, such as race. This was an important decision as women of colour are a double minority within CS and identifying participants as such could lead to their identification. The limitations related to this are discussed in Section 7.

Interviews were transcribed in full, and some initial themes were identified by one author. These themes were then discussed and developed between two authors, before all fifteen transcripts were thematically coded [8].

\section{FINDINGS}

\subsection{Similarities with other research}

Several of our findings mirror much other research into women's experiences in CS. Here, we briefly focus on two key themes ('male and geeky' stereotypes of CS and women's lack of confidence) that resonate with other research before we turn to the new and emerging themes we identified.

The 'male and geeky' stereotype': A gendered image of CS was common among participants, though they were aware this image was not necessarily accurate or helpful. When asked about the associations of the term 'computer science', P13 stated 'I always think of some sort of lonely guy in his office typing [...] some guy sat in his office in the dark at a weird time in the morning'. She continued: 'you do always think of men before you think of women when you think of the subject, well I do anyway. Just because of how it's been portrayed [in film and TV series] and what I've seen, you don't really think "oh it's going to be a bunch of girls in a room together"'. P9 also identified that 'when you think of computer science, it's more of like a guy behind a computer'. Whilst P8 identified CS as being about 'real world and real social problems', a mental image of CS as 'nerdy people in glasses sitting in a dark room' still prevailed. These perceptions of CS resonate with Sinclair and Kalvala's suggestion that CS is often seen as 'the domain of socially awkward men', innovation the result of 'individual males working alone [...] in little garages' [34]. The existence of such stereotypes has also been identified by Borsotti [7] and Volkel [37].

Lack of confidence: A lack of confidence has been reported in much of the literature on women in CS. This was echoed in our own findings, particularly demonstrated in the pervasive fear of falling behind. Our participants articulated many concerns around not keeping up or falling behind in future. Several participants felt that they were already behind, just 5-6 weeks into the course. P8, for example, stated 'I'm a bit behind at the moment', while P7 remarked 'I definitely am behind'. P6 echoed this, commenting that 'I feel like I'm falling behind a little bit', while P3 stated 'it is just sometimes tricky keeping up with everyone else'. For others, this was something they feared in the future. P5 voiced her fear of 'keeping up with everyone else' as the course progressed, whilst P2 commented 'I'm a bit nervous that I might fall behind if I don't really try my best to keep up with everything' and P11 remarked 'I'm nervous that I won't be able to keep up with work'.

Whilst this fear was partly connected to the intrinsic demands of the course, it was also related to the perceived greater experience and competency of 'everyone else' (P3, P5). Though P13 expressed the view that 'everyone's on the same level', many participants did not share this perspective. P8, for example, commented 'I think almost everyone I've met has had previous experience of coding', while P7 stated 'everyone else seems to have a background in Python'. P3 held the view that 'there are so many people who find this stuff easy' and are 'really good at it'. Such views were also held by participants with greater prior experience, such as CS A Level. P5, for example, identified that 'some of my friends who do computer science know quite a bit more than me', while P2 commented 'I know a lot of people have come into the degree with a lot more experience than I have'. P1 had programmed in C++ at school, but found it 'scary' when 'I started making friends and they are all talking about Python'. These findings resonate with other research that has discovered a lack of computing confidence in female students regardless of actual ability [5] [20] [27].

\subsection{New and emerging themes}

Our thematic analysis also identified two key themes that do not seem to have been considered by the diversity in CS literature and that represent key ways by which our participants navigated belonging uncertainty and being in the minority.

Navigating the minority experience - hedging: Several interview participants had experienced sexism or stereotyping in a CS context. Speaking a language with gendered nouns, for example, P11's CS teacher had used the male form of 'computer scientist' in class: 'it was... kind of not comfortable for me. Like, I'm sorry I'm not a boy!'. P14 had experienced her male peers' 'lad banter' in her 
Table 1: Sense of belonging card ranking exercise statements

\begin{tabular}{ll}
\hline Sense of belonging category & Statement \\
\hline Spaces/learning environment [31] & 'I feel comfortable in the computer labs' \\
Careers [26] & 'I think I would fit in at a tech company' \\
Creativity and design [32] & 'My course teaches me about computing's creative elements' \\
Society [18] & 'My course teaches me about how computing can solve social problems' \\
Terminology [21] & 'Terms like "computer science" are appealing to me' \\
Teaching diversity [33] & 'My course teaches me about the importance of considering diversity' \\
Community [10] & 'The department has a community that I feel part of' \\
Unconscious bias [22] & 'Language or examples used in the course have sometimes made me uncomfortable' \\
Making diversity visible [24] & 'I see people like me represented in computing' \\
\hline
\end{tabular}

CS classes, whilst P2 had felt discriminated against compared with her male CS A Level classmate: 'they'd always ask him if there was any technical help needed ... And then when he left, they never asked me, they'd go to an IT rep'.

However, despite the existence of negative experiences, the participants did not clearly state that they considered their minority situation a problem. Participants stressed that they were used to being in the minority and also demonstrated use of the linguistic device of hedging. Hedging involves the softening of statements by mitigators, qualifiers and diminishing clauses. Whilst subject to some debate, hedging has been found by some linguistic researchers to be more prevalent among women [11] [39].

Hedging is demonstrated in the ways in which participants narrated their experiences of sexism, being quick to diminish negative experiences. P13, having recounted a story of being treated with suspicion by a teaching assistant when she finished the assigned tasks early, commented: 'That might just be his personality, but it just came across a bit like - you know when you can tell someone doesn't believe that you've done this, or whether he's just like that anyway, but it was a bit like that wasn't fun. But I'm comfortable in all my other labs; that was just a particular experience where I was bit like "hmm" because everyone else had been so nice and so not like that, it came across as a bit strange [...] that was probably the worst thing that happened but other than that everyone seems to be fine. Like it was just this particular day, not good'. In this account, P13 oscillates frequently between articulating what had been a bad experience for her and trying to write off, explain, or diminish this experience. Finally, she turned the focus to her own expectations: 'I wasn't expecting that reaction [of "prove to me you've not cheated and you know what you're doing"], so it was a bit strange'.

Similarly to P13, P14 uses the word 'but' to diminish potentially negative experiences related to her male peers: 'I mean sometimes the lads are just annoying, 'cause they're immature - not immature towards me, but their jokes and stuff are just immature so I don't socialise with them outside of class, but they're not an issue'. She uses the same linguistic strategy when talking about a more clearly negative experience: 'they had quite a lot of lad banter and sometimes - not towards me, but like talking about other girls, like sexual comments, and it was a bit like "meh", but it wasn't too bad'.

Hedging was also demonstrated when respondents talked more generally about their experiences of the CS gender imbalance. P11 stated 'I expected that [the lack of equal gender representation].
I expected that a lot', but then commented 'it feels weird to be honest'. P11 also commented that it wasn't a problem but then subtly suggested it was something that she needed to get used to: 'I don't see it as a problem. Yeah, I think it's just the way it is. It's nothing to think much about. I mean yeah - like, I get used to it probably, so...' 'P3 also used hedging in her reflections on being one of a minority: 'sometimes I do wish there was more girls, because it's nice - I'm not saying like I hate sitting next to boys, because that's not true - like they're fine, it's good, but...'. P13 also again demonstrated this linguistic oscillation, commenting on the gender imbalance: 'it's never sort of annoyed me, 'cause I obviously get on with anyone, but it is a little bit - I don't know, I find it - it is a bit strange, but I think I guess I'm so used to it [... I I guess it's not really bothered me too much, but it is a bit weird sometimes'.

In these accounts, the participants never state explicitly that the situation they find themselves in is a problem, instead carefully couching their experiences using a linguistic device that serves to downplay their negative experiences or feelings: this can be summarised as 'it's a bit weird but it's ok'.

Individualisation of the problem: The second key theme we identify is a common individualisation of the problem. Firstly, our participants often interpreted questions about their experiences as women in CS in terms of their own personal, individual relationships with their male peers. There was generally a strong rhetoric of being able to make friends with their male peers, as P4 expressed: 'I can talk to guys, it's not a problem for me'. P13 commented similarly 'I obviously get on with anyone', while P10 echoed this: 'I got along with everyone in my class great anyway'. P8 expressed a similar sentiment: 'I think I get along with men and women completely equally, so I don't think I'd ever feel out of place, even if it was male-dominated'. By focusing on their own personal relationships with male peers, the minority situation is again diminished in our participants' accounts.

Secondly, our participants emphasised their own individual responsibility to fit in and succeed. Nerves and anxiety, for example, were seen as a personal problem to solve, as demonstrated by P3: 'when I go in [to the labs], I'm nervous about coding anyway. It's not like anyone's fault; it's just my own issues'. When considering how 'nerve-wracking' she found it to ask questions in the labs, she commented 'I need to put myself out there a bit more really', placing responsibility on herself. P11 was worried about making mistakes in the labs: 'sometimes I feel like I'm being judged by 
some people and I think it's probably not true and I'm hopefully wrong but I don't always feel comfortable. I sometimes feel like ... my friends even are like "oh ... she made a mistake" or something. Maybe it's just me'. She continued further 'I know it's not right to do that [worry about making mistakes], but still I can't help it'. Rather than any comments on the culture of the labs, P11 places responsibility on herself to change her mindset.

Whilst some participants had concerns about the workplace culture they might later face in the tech industry, they put emphasis on overcoming this to fit in. Having expressed concern that tech companies might have work meetings in the pub that excluded women, P10 explained that she would need to be: 'assertive enough to be like "ok I'm coming along". It is sort of difficult because they probably - people probably won't tell you, but ... I'd probably be confident to go up to people and ask them "oh yeah, is it happening or is it not happening?" and just like showing a certain level of confidence, because I've heard if you don't have that sort of confidence, you're going to get pushed out very, very easily. So I guess it would probably bother me a bit, but I would sort of try and push in and just involve myself, like throw myself into it'. Similarly P13, whilst slightly put off by media depictions of tech companies 'full of casually dressed men', stated that 'I'd make it work for myself. Like I wouldn't ever go in somewhere thinking "I'm not going to fit in". I'd go in somewhere being like "no, I belong, like I got here on my own credit, so I deserve this"'. P1 was more assertive in this view: 'if they [women] are interested in it, then they should not be scared to choose it because it's mainly males there'.

Finally, many participants placed emphasis on the issues of choice and access, rather than any broader, more societal, concerns pertaining to diversity in CS. Many participants spoke about women's choice to go into CS, as P1 explained: 'if women don't choose computer science and if they're not interested in it, then what's the problem? Like they're free to not choose it, as well as to choose it'. The solution for P1 was that 'everyone should be granted an access to try it and see if they like it but if they don't, that's completely fine'. P12 echoed this: 'I think if we gave all genders equal opportunity at the start, then gender balances further up the chain wouldn't be seen as an issue because nobody would even notice them. It would be about choice'.

However, though participants stressed individual choice to enter CS, there was some cynicism about CS diversity initiatives. P12 was told on her work experience that she had partly been chosen because she was female, and whilst she was 'happy it worked in my favour', she also expressed irritation that 'all the effort I put into my application didn't matter because I checked the box beside F'. P7 also felt frustrated when her head of sixth form ${ }^{3}$ told her that she'd have a good chance of getting into CS because 'they don't get many applications from women': she responded 'I'd really like it [uni application] to have a good chance, but at the same time I want it to be just for legitimate reasons'. P12 similarly resisted being 'prioritised just because of gender'. She continued that it was better for things to 'balance out naturally as opposed to feeling like we're pushing people in and out of courses just to make the gender

\footnotetext{
${ }^{3}$ Sixth form is the final two years of high school in the UK, at the end of which A Level exams are taken
}

balance "look better" because there's no point having more female computer scientists if they don't want to be doing comp sci'.

The idea of diversity as little more than a tick-box was only challenged by a small number of participants who articulated why diversity in CS might be important from a broader, societal perspective. P11 commented on the limits of teams of engineers who 'have the same experience in life [... and] all think the same way $[\ldots]$ and yeah, nothing new comes from there'. P10 similarly stated that 'if you've got people from different walks of life, they sort of have a different perspective on how they see things so they can kind of come up with problems they've experienced, and that can really have big social impact'. P14 also commented on how diversity 'brings more ideas to the table, more different perspectives [...] if they're making solutions that could go anywhere in the world then they should consider the other half of the population as being women'. It is worth noting that these three participants all referred to books they had read or podcasts they had listened to when talking about these issues. They had not learnt about this through their CS studies, but through their own learning and pursuit of personal interests outside of formal educational contexts.

\section{DISCUSSION AND IMPLICATIONS}

Digital knowledge and skills are more relevant and important than ever for current and future careers, increasingly seen as essential for young people to participate fully, and safely, in our constantly evolving digital economy and society. However, there are major challenges in realising this. Beyond the rhetorical position that 'computing is for everyone', the discipline continues to be beset by a lack of diversity at all levels, through school, university and careers. This situation is concerning in and of itself - as a lack of diversity may discourage more marginalised groups from entering the field - but also brings with it broader societal and ethical concerns, such as gender and racial biases in app design and algorithms [25].

In this paper, we report on the findings of 15 semi-structured interviews with first year female CS undergraduate students. Our interviews confirm other research findings regarding prevalent stereotypes of CS as 'male and geeky' and women experiencing a lack of confidence in CS contexts. In these ways not much has changed since the publication of Unlocking the Clubhouse [21]. However, we also identify two key themes that are under-explored in the literature. By hedging and individualising the problem, female CS students seem to have developed two key mechanisms of resilience, forming a 'way of being' - a means to justify their place in a predominantly male CS education environment.

However, it should not make comfortable reading that female undergraduate CS students linguistically mitigate and diminish their more negative experiences of CS culture as something 'strange', 'weird', 'annoying' and 'not comfortable'. The intentionality in the voices of these women and their positivity in the face of difficulties is truly admirable, but points to the ongoing need for individuals to 'paper over' an underlying problem. The issue of hedging is a complex one, with much linguistic debate around its functions; whether, for example, it expresses tentativeness or, interpreted more positively, it is in fact a linguistic style more open to dialogue and shared communication [11] [39]. However, it is striking that hedging was used so frequently when the issue of gender imbalance was brought 
up. Whilst Walton and Cohen's concept of 'belonging uncertainty' refers to uncertainty regarding social connections [38], we suggest that this concept can be extended to refer to how female CS students verbally articulate their experiences, their reflections on the gender imbalance of CS being characterised by uncertainty. This suggests a complex and fragile sense of belonging experienced by women as a minority group in CS, especially when considered alongside our participants' awareness of strongly gendered CS stereotypes and their widespread fear of falling behind.

Our data also highlights that participants embrace an individualistic perspective that stresses the importance of choice and of self-responsibility to fit in and succeed. Whilst we are not aware of literature in CS that has highlighted this, this phenomenon has been researched in youth studies, with one study demonstrating that, despite experiences of structural inequality, their participants exhibited 'a deep-seated faith in the power of hard work to overcome obstacles' and 'a self-focused approach to process dissonant information and experiences' [14]. This has clear resonance with our own findings. Whilst it is encouraging to hear that our participants had a strong sense of their own personal agency, we argue that responsibility needs to extend beyond individuals, with educational and work contexts being also responsible for creating inclusive cultures. The rhetoric of individual responsibility also has a flipside, as our findings demonstrate. Whilst our participants expressed confidence in their own ability to succeed, when they did experience setbacks, they placed blame upon themselves. This raises questions regarding the fragility of this discourse of individual responsibility in the context of ongoing gender imbalance within CS.

Our findings have significant implications for CS educators, presenting us with an urgent challenge in our education systems to understand how to foster a stronger sense of belonging for women in CS. This is a multi-dimensional challenge incorporating many different aspects. Our participants' accounts suggest that attention needs to be given to challenging stereotypes of CS that remain worryingly prevalent. Making diversity visible - that is, drawing attention to and celebrating the contributions of a diverse range of people within CS - offers one way forward to disrupt the 'male and geeky' stereotype. Stereotypes of CS also raise questions as to how CS is defined as a discipline, including where CS is perceived to sit on various different spectra (Figure 1), when considering our lived culture and practices, and the design of our courses and materials. In addition, attention needs to be given to how physical and online teaching spaces are designed, and specifically how to create spaces where mistakes and questions are welcomed.

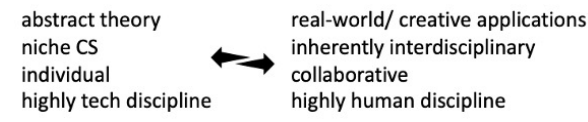

Figure 1: Different spectra

The emphasis young women place on their own individual responsibility presents challenges to CS departments, institutions, and the discipline as a whole. We advocate that there is a need to understand and externalise the underpinning social and structural aspects of our CS educational practices and remove restrictive barriers for minority groups. The small number of participants who were aware of the wide-ranging societal implications of a homogeneous tech industry had learnt about this outside of their formal CS education. This suggests that more discussion of these issues within formal CS teaching could provide young women with a vocabulary to greater articulate and make sense of their experiences, as well as ensuring that all CS students learn about ethical issues related to technology design and development.

\section{LIMITATIONS AND FUTURE RESEARCH}

There are three key limitations to this study. Firstly, this study relied upon a volunteer sample, raising questions about who chose to volunteer and who did not. An initial concern, however, that more gender-aware or feminist-identifying young women might be more likely to volunteer proved unfounded, as our interviewees expressed a wide range of perspectives. Secondly, we did not interview male students. Whilst this would have offered a useful comparison, we specifically wanted to foreground young women's experiences and voices. Future avenues of research include conducting a survey of all CS students to compare male and female students' sense of belonging. Finally, our interviews were conducted early on in the academic year, when the participants were new to university. This newness may have influenced some of their responses. Further longitudinal work will be valuable in seeing how these perspectives and attitudes might change over time, and as women progress in their studies. This work is part of a larger project, which has also conducted surveys with female CS A Level students. Future work will identify differences and similarities between students at $\mathrm{A}$ Level and undergraduate level. It is also worth commenting that concern for our participants' anonymity made it challenging for us to fully draw out the intersectional nature of their experiences, these experiences being not only gendered but shaped by other demographic variables, such as race. In the interest of protecting our participants, we do not report on these additional variables, but this does unfortunately omit aspects of our participants' lived experiences.

\section{CONCLUSIONS}

This paper, drawing on interviews with fifteen first year female undergraduates, reveals a complex picture of how young women negotiate being in a minority in CS and their sense of belonging. Our participants exhibited two key strategies for navigating their experiences. Firstly, they used the linguistic device of hedging to mitigate or diminish negative experiences or feelings; secondly, they placed a high degree of emphasis on their own individual responsibility to succeed and fit in. We argue that hedging serves to express uncertainty regarding belonging, suggesting - when taken into consideration alongside gendered stereotypes of CS and fears of falling behind - that women's sense of belonging in CS may be fragile. Whilst a strong sense of personal agency is to be welcomed, this emphasis on individual responsibility may also lead young women to blame themselves for negative experiences. There are no easy solutions to these complex experiences of being a minority, but clearly much work is needed to consider how to respond and how to foster a more robust and resilient sense of belonging for women within CS. 


\section{REFERENCES}

[1] Christine Alvarado, Yingjun Cao, and Mia Minnes. 2017. Gender Differences in Students' Behaviors in CS Classes throughout the CS Major. In Proceedings of the 2017 ACM SIGCSE Technical Symposium on Computer Science Education (Seattle, Washington, USA) (SIGCSE '17). Association for Computing Machinery, New York, NY, USA, 27-32. https://doi.org/10.1145/3017680.3017771

[2] atomico. 2020. The State of European Tech 2020. Retrieved 13 Jan 2021 from https://2020.stateofeuropeantech.com/chapter/diversity-inclusion/article/ call-to-action/

[3] Catherine Ball, Faron Moller, and Reena Pau. 2012. The Mindstorm Effect: A Gender Analysis on the Influence of LEGO Mindstorms in Computer Science Education. In Proceedings of the 7th Workshop in Primary and Secondary Computing Education (Hamburg, Germany) (WiPSCE '12). Association for Computing Machinery, New York, NY, USA, 141-142. https://doi.org/10.1145/2481449.2481483

[4] BCS. 2020. BCS Virtual Insights 2020. Retrieved 13 Jan 2021 from https://www. bcs.org/media/5873/virtual-insights-2020-report.pdf

[5] Sylvia Beyer, Kristina Rynes, Julie Perrault, Kelly Hay, and Susan Haller. 2003 Gender Differences in Computer Science Students. SIGCSE Bull. 35, 1 (Jan. 2003), 49-53. https://doi.org/10.1145/792548.611930

[6] Jennifer M. Blaney and Jane G. Stout. 2017. Examining the Relationship Between Introductory Computing Course Experiences, Self-Efficacy, and Belonging Among First-Generation College Women. In Proceedings of the 2017 ACM SIGCSE Technical Symposium on Computer Science Education (Seattle, Washington, USA) (SIGCSE '17). Association for Computing Machinery, New York, NY, USA, 69-74. https://doi.org/10.1145/3017680.3017751

[7] Valeria Borsotti. 2018. Barriers to Gender Diversity in Software Development Education: Actionable Insights from a Danish Case Study. In Proceedings of the 40th International Conference on Software Engineering: Software Engineering Education and Training (Gothenburg, Sweden) (ICSE-SEET '18). Association for Computing Machinery, New York, NY, USA, 146-152. https://doi.org/10.1145/ 3183377.3183390

[8] Virginia Braun and Victoria Clarke. 2006. Using Thematic Analysis in Psychology. Qualitative Research in Psychology 3, 2 (2006), 77-101.

[9] Mathilde Collain and Deborah Trytten. 2019. "You Don't Have to Be a White Male That Was Learning How to Program since He Was Five". In Proceedings of the 50th ACM Technical Symposium on Computer Science Education (Minneapolis, MN, USA) (SIGCSE '19). Association for Computing Machinery, New York, NY, USA, 968-974. https://doi.org/10.1145/3287324.3287383

[10] Wendy M. DuBow, Beth A. Quinn, Gloria Childress Townsend, Rosario Robinson, and Valerie Barr. 2016. Efforts to Make Computer Science More Inclusive of Women. ACM Inroads 7, 4 (Nov. 2016), 74-80. https://doi.org/10.1145/2998500

[11] Penelope Eckert and Sally McConnell-Ginet. 2013. Language and Gender (2 ed.) Cambridge University Press. https://doi.org/10.1017/CBO9781139245883

[12] Katrina Falkner, Claudia Szabo, Dee Michell, Anna Szorenyi, and Shantel Thyer. 2015. Gender Gap in Academia: Perceptions of Female Computer Science Academics. In Proceedings of the 2015 ACM Conference on Innovation and Technology in Computer Science Education (Vilnius, Lithuania) (ITiCSE '15). Association for Computing Machinery, New York, NY, USA, 111-116. https: //doi.org/10.1145/2729094.2742595

[13] Joint Council for Qualifications. 2020. A Level and AS Results Summer 2020 Retrieved 13 Jan 2021 from https://www.jcq.org.uk/wp-content/uploads/2020/ 09/A-Level-and-AS-Results-Summer-2020.pdf

[14] Michela Franceschelli and Avril Keating. 2018. Imagining the Future in the Neoliberal Era: Young People's Optimism and Their Faith in Hard Work. YOUNG 26, 4_suppl (2018), 1S-17S. https://doi.org/10.1177/1103308817742287

[15] Michail N. Giannakos, Letizia Jaccheri, and Roberta Proto. 2013. Teaching Computer Science to Young Children through Creativity: Lessons Learned from the Case of Norway. In Proceedings of the 3rd Computer Science Education Research Conference on Computer Science Education Research (Arnhem, Netherlands) (CSERC '13). Open Universiteit, Heerlen, Heerlen, NLD, 103-111.

[16] HESA. 2020. What do HE students study? Personal characteristics, 2018-19 data. Retrieved 13 Jan 2021 from https://www.hesa.ac.uk/data-and-analysis/students/ what-study/characteristics

[17] Nicolas Kayser-Bril. 2020. Female historians and male nurses do not exist, Google Translate tells its European users. Retrieved 13 Jan 2021 from https: //algorithmwatch.org/en/story/google-translate-gender-bias/

[18] Vivian Anette Lagesen. 2007. The Strength of Numbers: Strategies to Include Women into Computer Science. Social Studies of Science 37, 1 (2007), 67-92.

[19] Karyn L. Lewis, Jane G. Stout, Noah D. Finkelstein, Steven J. Pollock, Akira Miyake, Geoff L. Cohen, and Tiffany A. Ito. 2017. Fitting in to Move Forward: Belonging, Gender, and Persistence in the Physical Sciences, Technology, Engineering, and Mathematics (pSTEM). Psychology of Women Quarterly 41, 4 (2017), 420-436.

[20] Alex Lishinski, Aman Yadav, Jon Good, and Richard Enbody. 2016. Learning to Program: Gender Differences and Interactive Effects of Students' Motivation, Goals, and Self-Efficacy on Performance. In Proceedings of the 2016 ACM Conference on International Computing Education Research (Melbourne, VIC, Australia) (ICER '16). Association for Computing Machinery, New York, NY, USA, 211-220. https://doi.org/10.1145/2960310.2960329

[21] Jane Margolis and Allan Fischer. 2002. Unlocking the Clubhouse: Women in Computing. MIT Press, Cambridge, MA, USA.

[22] Paola Medel and Vahab Pournaghshband. 2017. Eliminating Gender Bias in Computer Science Education Materials. In Proceedings of the 2017 ACM SIGCSE Technical Symposium on Computer Science Education (Seattle, Washington, USA) (SIGCSE '17). Association for Computing Machinery, New York, NY, USA, 411-416. https://doi.org/10.1145/3017680.3017794

[23] Allison Mishkin. 2019. Applying Self-Determination Theory towards Motivating Young Women in Computer Science. In Proceedings of the 50th ACM Technical Symposium on Computer Science Education (Minneapolis, MN, USA) (SIGCSE '19). Association for Computing Machinery, New York, NY, USA, 1025-1031. https://doi.org/10.1145/3287324.3287389

[24] Alejandra Montoya. 2017. Computer Science for All: Opportunities Through a Diverse Teaching Workforce. Harvard fournal of Hispanic Policy 29 (2017), 47 62.

[25] Caroline Criado Perez. 2019. Invisible Women: Exposing Data Bias in a World Designed for Men. Chatto and Windus.

[26] Abigail Powell, Andrew Dainty, and Barbara Bagilhole. 2012. Gender Stereotypes Among Women Engineering and Technology Students in the UK: Lessons from Career Choice Narratives. European fournal of Engineering Education 37, 6 (2012), 541-556. https://doi.org/10.1080/03043797.2012.724052

[27] Keith Quille, Natalie Culligan, and Susan Bergin. 2017. Insights on Gender Differences in CS1: A Multi-Institutional, Multi-Variate Study.. In Proceedings of the 2017 ACM Conference on Innovation and Technology in Computer Science Education (Bologna, Italy) (ITiCSE '17). Association for Computing Machinery, New York, NY, USA, 263-268. https://doi.org/10.1145/3059009.3059048

[28] Yolanda A. Rankin and Jakita O. Thomas. 2020. The Intersectional Experiences of Black Women in Computing. In Proceedings of the 51st ACM Technical Symposium on Computer Science Education (Portland, OR, USA) (SIGCSE '20). Association for Computing Machinery, New York, NY, USA, 199-205. https://doi.org/10.1145/ 3328778.3366873

[29] Katie Redmond, Sarah Evans, and Mehran Sahami. 2013. A Large-Scale Quantitative Study of Women in Computer Science at Stanford University. In Proceeding of the 44th ACM Technical Symposium on Computer Science Education (Denver, Colorado, USA) (SIGCSE '13). Association for Computing Machinery, New York, NY, USA, 439-444. https://doi.org/10.1145/2445196.2445326

[30] Mike Sands, Nick Moukhine, and Glenn Blank. 2008. Widening the Pipeline of K-12 Students with Flash. f. Comput. Sci. Coll. 23, 5 (May 2008), 52-57.

[31] Paul G. Davies Sapna Cheryan, Victoria C. Plaut and Claude M. Steele. 2009. Ambient Belonging: How Stereotypical Cues Impact Gender Participation in Computer Science. Fournal of Personality and Social Psychology 97, 6 (2009), $1045-1060$.

[32] Linda J. Sax, Kathleen J. Lehman, Jerry A. Jacobs, M. Allison Kanny, Gloria Lim, Laura Monje-Paulson, and Hilary B. Zimmerman. 2017. Anatomy of an Enduring Gender Gap: The Evolution of Women's Participation in Computer Science. The Journal of Higher Education 88, 2 (2017), 258-293.

[33] Kimberly Scott, Gregory Aist, and Xiaolong Zhang. 2014. Designing a Culturally Responsive Computing Curriculum for Girls. International fournal of Gender, Science and Technology 6, 2 (2014), 264-276.

[34] Jane Sinclair and Sara Kalvala. 2015. Exploring Societal Factors Affecting the Experience and Engagement of First Year Female Computer Science Undergraduates. In Proceedings of the 15th Koli Calling Conference on Computing Education Research (Koli, Finland) (Koli Calling '15). Association for Computing Machinery, New York, NY, USA, 107-116. https://doi.org/10.1145/2828959.2828979

[35] Jessi L. Smith, Karyn L. Lewis, Lauren Hawthorne, and Sara D. Hodges. 2013. When Trying Hard Isn't Natural: Women's Belonging With and Motivation for Male-Dominated STEM Fields As a Function of Effort Expenditure Concerns. Personality and Social Psychology Bulletin 39, 2 (2013), 131-143.

[36] Jane G. Stout, N. Burçin Tamer, and Christine J. Alvarado. 2018. Formal Research Experiences for First Year Students: A Key to Greater Diversity in Computing?. In Proceedings of the 49th ACM Technical Symposium on Computer Science Education (Baltimore, Maryland, USA) (SIGCSE '18). Association for Computing Machinery, New York, NY, USA, 693-698. https://doi.org/10.1145/3159450.3159472

[37] Sarah Theres Völkel, Wiktoria Wilkowska, and Martina Ziefle. 2018. GenderSpecific Motivation and Expectations toward Computer Science. In Proceedings of the 4th Conference on Gender \& IT (Heilbronn, Germany) (GenderIT '18). Association for Computing Machinery, New York, NY, USA, 123-134. https://doi.org/10.1145/3196839.3196858

[38] Gregory M. Walton and Geoffrey L. Cohen. 2007. A Question of Belonging: Race, Social Fit, and Achievement. Fournal of Personality and Social Psychology 92, 1 (2007), 82-96.

[39] Ann Weatherall. 2005. Gender, Language and Discourse. Routledge.

[40] David C. Webb, Alexander Repenning, and Kyu Han Koh. 2012. Toward an Emergent Theory of Broadening Participation in Computer Science Education. In Proceedings of the 43rd ACM Technical Symposium on Computer Science Education (Raleigh, North Carolina, USA) (SIGCSE '12). Association for Computing Machinery, New York, NY, USA, 173-178. https://doi.org/10.1145/2157136.2157191 